\title{
Surgical Removal of Complex Odontoma - Case Report
}

\author{
Dr Naveen Krishna Kv ${ }^{1}$, Dr Akshata. G. Kamath ${ }^{2}$, Dr Subhashini. $\mathrm{S}^{3}$, \\ Dr Renjith Madhavan ${ }^{4}$ \\ ${ }^{\text {I}}$ (Professor / Periodontics / Century International Institute Of Dental Sciences \\ And Research Centre/India) \\ ${ }^{2,4}$ (Senior Lecturer /Periodontics/ Century International Institute Of Dental Sciences \\ And Research Centre / India \\ ${ }^{3}$ (Reader/ Orthodontics /Century International Institute Of Dental Sciences \\ And Research Centre ,India)
}

\begin{abstract}
Odontomas are hamartomatous malformations of odontogenic origin. During tooth developmental stages any defect in the maturation phase of morpho-differentiation leads to such anomalies. Odontomas are benign, slow-growing and nonaggressive clinically asymptomatic and are diagnosed on routine radiological examination. In our case report an asymptomatic hard growth in the upper right anterior region of the maxilla associated with impacted canine in a 16-year-old female was reported, panoramic radiograph revealed amorphous mass of calcified material with the radiodensity of tooth structure, with no anatomical resemblance to tooth, surrounded by a narrow radiolucent rim, suggestive of complex odontoma. Under local anesthesia, access to the lesion was achieved via intra-oral approach and surgical excision. To enable the eruption of retained permanent canine in order to establish harmony in the development of dental arch .
\end{abstract}

Keywords: complex odontoma, hamartoma, odontogenic tumor, orthopantomogram

\section{Introduction}

Odontomas refer to tumors of odontogenic origin. ${ }^{[1]}$ Paul Broca in 1867 coined the term odontoma , referring to tumors formed by the overgrowth of transient or complete dental tissues. ${ }^{[2],[3]}$ Through usage they are considered to be hamartomatous malformation rather than true neoplasm. ${ }^{[4]}$ The World Health Organization, in 2005 categorized odontomas as calcified irregular mass not resembling teeth, as complex odontome and calcified mass bearing resemblance to the teeth, as compound odontome ${ }^{[5]}$ Complex odontomas occur in the posterior mandible and compound odontomas are more common in the anterior maxilla. ${ }^{[2],[4],[7]}$ Complex odontomas are less common than the compound variety in the ratio $1: 2{ }^{[2],[7],[8]}$ Odontomas are usually asymptomatic and appear as small solitary symptomless lesions, most associated with unerupted teeth found on routine radiographic examination. ${ }^{[9]}$.

\section{Case Report}

A 16-year-old female patient reported to the dental clinic with a complaint of swelling in the upper right anterior segment since 6 months. Intraoral examination revealed a swelling on the alveolar ridge distal to right maxillary lateral incisor (Figure 1). On palpation the mass was non-tender and hard in consistency with an expansion of buccal cortical bone the contra lateral teeth (maxillary canine ) had not erupted.

Radiographic examination using orthopantamogram ( Fig 2 ) revealed a well demarcated radio opaque lesion, with radiodensity as that of a tooth interspersed with radiolucent areas surrounded by a radiolucent rim distal to the right maxillary lateral incisor and mesial to maxillary first premolar area, coronal to the retained permanent canine. Based on the above finding a diagnosis of complex odontoma was made.

Intraoraly the lesion painted with $5 \%$ povidone iodine solution following which local anesthesia was administered . A semilunar incision given in relation to upper anterior vestibule extending from midbuccal aspect of 11 to midbuccal aspect of 14. After full thickness mucoperiosteal flap was raised, bunt dissection was done bony protuberance was observed Superficial bone was removed with round bur, conglomerate mass of ill formed calcified structures were removed with elevator without damage to adjacent teeth (Fig 3). Flap was approximated and sutured with 3-0 silk suture. Histopathological examination of the decalcified hard tissue mass revealed an ill-organized structure of enamel, dentin, cementum and pulp tissue. With larger quantities dentine and cementum. One week post operative, recovery was uneventful (Fig 4). 


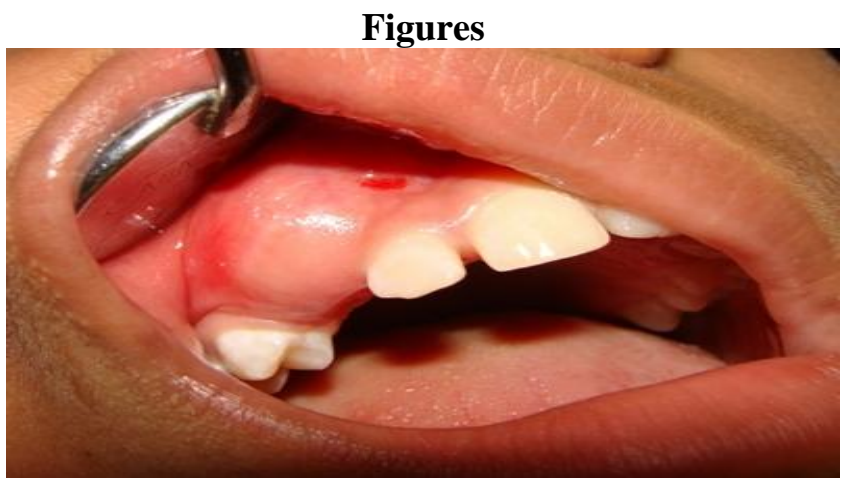

"Fig 1: Intraoral swelling in the upper right anterior segment"

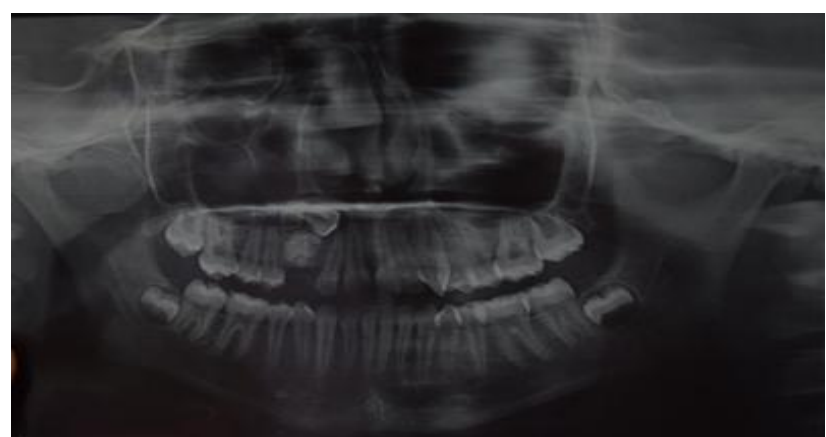

"Fig 2- orthopentamogram showing the lesion"

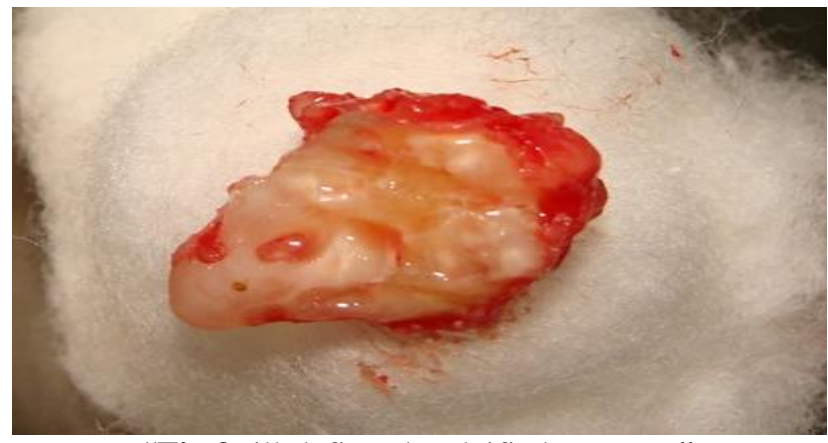

"Fig 3: ill-defiened calcified structure"

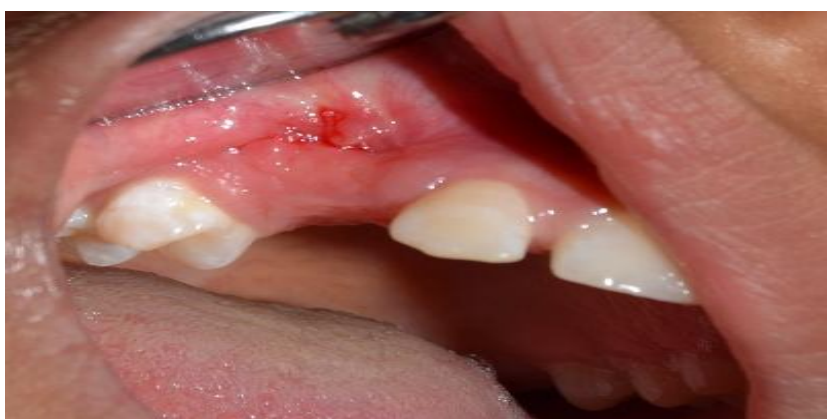

"Fig 4: one week post healing"

\section{Discussion}

Mc Kinney 1984; Gomel and Seckin, 1989 have suggested that odontoma is a tumor formed by dental structures (enamel, dentine pulp and cement), which accounts for $22 \%$ of the odontogenic tumours. ${ }^{[10]}$, [9], [11] However exact cause is unknown , possibility includes trauma to primary dentition, inflammatory and infectious processes, odontoblastic hyperactivity, mutant gene. $\left.{ }^{[6}\right],{ }^{[12]}$ and hereditary anomalies such as Gardner's syndrome and Hermann's syndrome . ${ }^{[13]}$ During the developmental stages if a portion of dental lamina persists results formation of compound or complex odontoma. ${ }^{[6]}$ 
Radiographs are a definitive aid in the diagnosis of odontomas. ${ }^{[9]}$ In our case the lesion appeared as an irregular radiodense calcified mass surrounded by a thin radiolucent area, with no resemblance to dental structures ${ }^{[1],[2]}$ confirmatory to our diagnosis of complex odontoma. Radiographs also aid in assessing the development stages of odontoma formation based on the degree calcification. In the initial stage the lesion appears radiolucent due to absence of calcification; intermediate stage is characterized by partial calcification; and in the final stage the odontoma appears radiopaque surrounded by a radiolucent halo. ${ }^{[7],[8]}$ Since odontomas are well capsulated lesions and have less chances of recurrence, the management comprises of a conservative surgical excision. ${ }^{[10],[14]}$

Although not aggressive exhibits limited growth potential, early diagnosis is very important so as to establish harmony in the development of dental arch and occlusion including esthetics. ${ }^{[10]}$

\section{Conclusion}

Odontomas are odontogenic tumors composed of dental tissues. They are slow-growing, benign tumours showing nonaggressive behaviour usually associated with unerupted tooth, usually detected incidentally on routine radiographic examination. Timely detection and surgical correction is required to prevent complications such as , cystic changes, bone expansion, and delayed eruption.

\section{References}

[1]. Shafer W.G , Hine M. K, and Levy B.M "Cysts and tumours of the jaws," in A Textbook of Oral Pathology, pp. 308-311, WB Saunders, Philadelphia, Pa, USA, 4th edition, 1997.

[2]. Cohen DM., Bhattacharyya I . "Ameloblastic fibroma, ameloblastic fibro-odontoma, and odontoma." Oral and Maxillofacial Surgery Clinics .2004; 16 ( 3): 375-384.

[3]. Sarojini S B ,Khosla E, Varghese T, and Arakkal LJ, "Eruption of Odontomas into the Oral Cavity: A Report of 2 Cases," Case Reports in Dentistry, vol. 2014, Article ID 639173, 4 pages, 2014. doi:10.1155/2014/639173

[4]. Neville BW, Damm DD, Allen CM, Bouquot JE. Oral \& Maxillofacial Pathology. 2nd ed. Philadelphia: Saunders; 2002. p. 631-2.

[5]. Morgan P. Odontogenic tumors: a review. Periodontology 2000. 2011; 57:160-76.

[6]. Shunmugavelu K, Subramaniam K . "Compound Composite Odontome In A 9 Year Old Female In Anterior Maxillary Alveolus Region: A Case Report" Int J Dent Health Sci 2015; 2(4): 1022-1026

[7]. Vengal M, Arora H, Ghosh S, Pai KM. "Large Erupting Complex Odontoma: A Case Report”. J Can Dent Assoc 2007;73:169-73

[8]. Patil S, Ramesh D, Kalla AR ."Complex Odontoma: Report Of Two Unusual Cases” Braz J Oral Sci.2012 ;11 (4) : $509-512$.

[9]. Das UM, Nagarathna, Arathi. "Unerupted Maxillary Canine Associated With Compound Composite Odontoma: A Case Report”. J Indian Soc Pedo Prev Dent. $2002 ; 20(3)$ : 98-101

[10]. Toledo OA, Bormann EP, Bezerra ACB . "Odontomas-Report Of 3 Cases" Braz Dent J 1992 3: 59-66.

[11]. Amado CS, Gargallo AJ, Berini AL, Gay EC. Review of 61 cases of odontoma. Presentation of an erupted complex odontoma. Medicina oral: organo oficial de la Sociedad Espanola de Medicina Oral y de la Academia Iberoamericana de Patologia y Medicina Bucal. 2002 Dec;8(5):366-73. 